\title{
Performance of the ATLAS tau-lepton trigger at the LHC in Run 2
}

\author{
Serhat Ördek*, on behalf of the ATLAS Collaboration \\ II. Physikalisches Institut, Georg-August-Universität Göttingen \\ E-mail: serhat.oerdekecern.ch
}

The ATLAS experiment has a rich physics programme of Standard Model measurements and searches for physics beyond the Standard Model involving tau-leptons. Most of these analyses depend on an efficient tau-lepton trigger that can cope with the overwhelming background from multi-jet events produced in proton-proton collisions at the Large Hadron Collider. The ATLAS trigger system is composed of two stages. At Level-1, tau leptons are reconstructed as energy deposits in neighbouring towers of calorimeter cells. The High Level Trigger (HLT) exploits the full calorimeter granularity as well as inner-detector tracks, and runs reconstruction and identification algorithms similar to those used in the offline reconstruction. The performance of the tau-lepton trigger in ATLAS Run 2 data will be discussed, and trigger efficiencies measured with a tag-and-probe method will be presented. An emphasis will be made on the improved HLT algorithms deployed in 2018. The association of tracks to the energy deposit in the calorimeter was tightened to reduce the contamination from fake tracks at high pile-up. An energy calibration based on a Boosted Regression Tree with improved energy resolution has replaced the simpler calibration based on pileup subtraction and calorimeter response correction. An identification algorithm based on a Recurrent Neural Network was also deployed, which provides increased jet rejection compared to the previously-used Boosted Decision Tree identification algorithm.

European Physical Society Conference on High Energy Physics - EPS-HEP2019 -

10-17 July, 2019

Ghent, Belgium

${ }^{*}$ Speaker. 


\section{Introduction}

A large part of the physics programme of the ATLAS collaboration [1] relies on the efficient reconstruction of hadronically decaying tau-leptons. They are used for measurements within the Standard Model, such as the observation of the Higgs boson coupling to tau-leptons [2], and searches for physics beyond the Standard Model, such as the search for pair-production of tausleptons [3].

But since the large event rate at the LHC in Run 2 makes it impossible to store all collision events for analyses like these, the trigger system is needed. This system comprises a variety of fast algorithms searching for specific properties in the events, to decide whether to keep or discard the event information permanently. This report covers the strategy and performance of the trigger algorithms used by ATLAS in Run 2 to identify events where hadronic tau-lepton decays occur.

\section{Tau-trigger strategy}

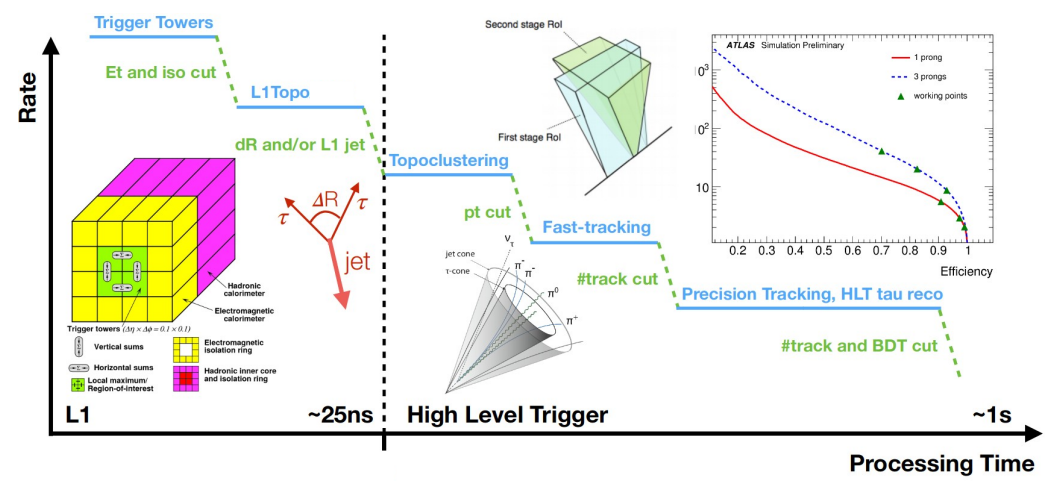

Figure 1: Graphical representation of the composition of an ATLAS tau-trigger chain [4].

Tau-leptons have a mass of $1.777 \mathrm{GeV}$ and a decay length of $87 \mu \mathrm{m}$, thus most decays occur before reaching active detector material. They decay into either light leptons or hadrons. In the $65 \%$ of cases where the tau-lepton decays hadronically, they must be identified by a tau-trigger, which is challenging in the hadron-dominated LHC environment, especially with the limited computational resources at trigger-level.

The method applied to identify these is depicted in Figure 1. The Run 2 trigger system is split into the Level-1 (L1) trigger and the High Level Trigger (HLT). As all of the approximately 40 million events per second of operation must be processed by L1, track information cannot be used there, as reconstructing tracks would take too long.

Instead, narrow energy deposits are selected in the electromagnetic and hadronic calorimeters and cuts are applied to the total energy [4]. In some cases, a subsequent cut on the energy detected around the main energy deposit is applied, to reject the main background of quark- or gluoninitiated jets. Optionally, topological cuts such as angular separation requirements or overlap removals can be applied between the tau candidates and other objects like jets or electrons.

The accepted events are processed by the HLT with a more accurate estimation of position and 


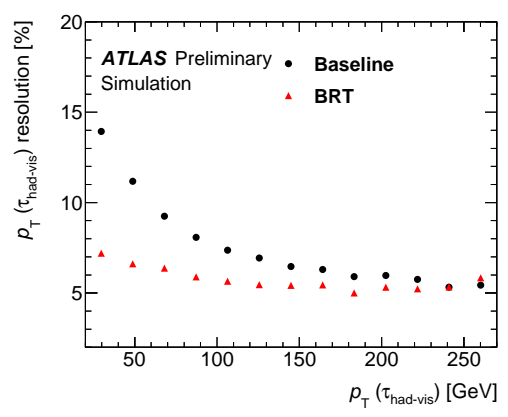

Figure 2: Comparison of the transverse momentum resolution as a function of the transverse momentum $p_{\mathrm{T}}$ of tau candidates using the baseline and BRT energy calibration [4].

energy. The first step in the HLT tau reconstruction chain, the calorimeter-only preselection, is based on clustering the calorimeter energy deposits assigned to the L1 tau candidate. Afterwards, a cut on its energy, calibrated as described in Section 3, is applied. Using a fast track reconstruction algorithm, the tau candidate is assigned tracks in a core and an isolation region surrounding it. The tau-lepton candidate is required to have 1 to 3 core tracks and less than 2 isolation tracks. The latest tau-triggers additionally accept tau candidates without any core tracks. Afterwards, the track reconstruction is improved with a more efficient algorithm based on the one used offline [5], and the track multiplicity cuts are re-applied. Finally, the tau identification described in Section 4 is used to select the accepted tau-lepton candidate events.

\section{Energy calibration}

Over the course of Run 2, the tau-lepton energy calibration in the calorimeter-only preselection of the HLT has changed. The baseline method was based on first subtracting any pile-up contribution to the calorimeter response and then applying correction factors determined using simulated events. To improve performance, a boosted regression tree (BRT) was trained to estimate the correct $p_{\mathrm{T}}$ value based on information such as the uncalibrated tau $p_{\mathrm{T}}$ and associated calorimeter cluster moments, leading to the improvement in energy resolution shown in Figure 2. A similar development occurred in the offline tau-lepton reconstruction [6]. The main difference is that only the offline version distinguishes between candidates with one or three associated tracks (called 1- or 3prong tau candidates), since track information is not available at the calorimeter-only preselection stage of the HLT reconstruction, where this calibration is applied.

\section{RNN-based identification}

Like the energy calibration, the HLT tau identification algorithm has improved during Run 2. A recurrent neural network (RNN) was trained to replace the boosted decision tree (BDT) that suffers from a drop in efficiency in events with higher pile-up. As shown in Figure 3, the efficiency has improved significantly while the rate is almost unchanged, which means that the background rejection has improved as well. The RNN is described in more detail in Ref. [8]. 


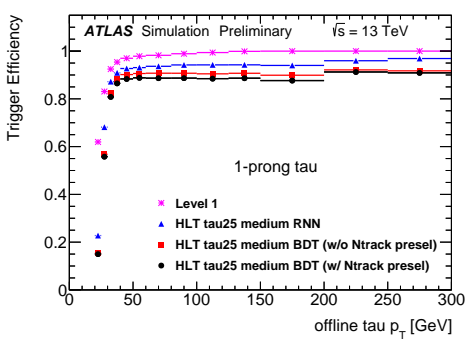

(a)

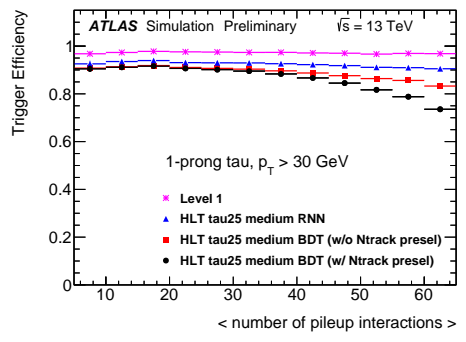

(b)

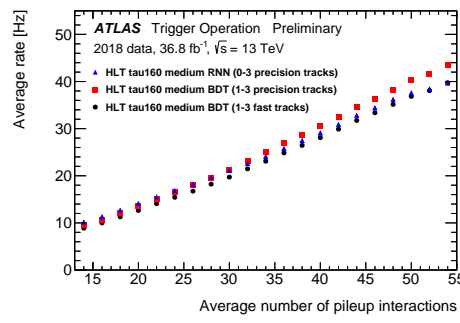

(c)

Figure 3: Comparison of trigger chains using either the BDT or the RNN identification [7]. The black markers show the performance of the trigger chains used since the start of Run 2. For the red and blue markers, no cut is applied after the first track reconstruction.

\section{Tau trigger menu}

The ATLAS trigger menu contains many tau-trigger chains targeting different final states. This is necessitated by the large QCD background, which, despite the advanced triggering techniques used, makes it difficult to achieve reasonable event rates. To lower the rates, multi-object triggers with taus are used, which can then be operated with lower energy thresholds. As an example, the energy threshold for the single-tau trigger in 2018 was $160 \mathrm{GeV}$, whereas the di-tau trigger thresholds were 80 and $60 \mathrm{GeV}$. Thus, the varied tau-trigger menu helps cover different event topologies without incurring too high rates.

\section{Trigger efficiency measurement}

The efficiency of tau-triggers, as shown for example in Figure 4, is measured in an analysis requiring one muon and one hadronic tau-lepton decay candidate per event, designed to achieve a high purity of $Z \rightarrow \tau \tau$ events. However, tau-leptons from such events rarely reach high $p_{\mathrm{T}}$ values, making it difficult to estimate the efficiencies of trigger chains with high $p_{\mathrm{T}}$ thresholds. To that end, complementary analyses using $t \bar{t}$ and $W \rightarrow \tau \nu_{\tau}$ events were implemented.

\section{Development for Run 3}

For Run 3, an improved L1 calorimeter trigger will be used [9], granting better spatial resolution and thus better background rejection. The energy resolution will also improve. This is especially important for tau-triggers, as their main inefficiency in Run 2 came from the L1 energy cut.

\section{Conclusion}

The tau-trigger chains used within ATLAS have changed in various ways throughout Run 2 in order to cope with the increase in pile-up. The HLT tau energy calibration currently uses a 


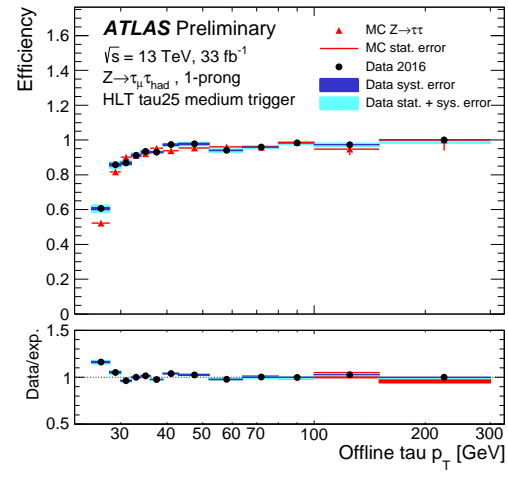

(a)

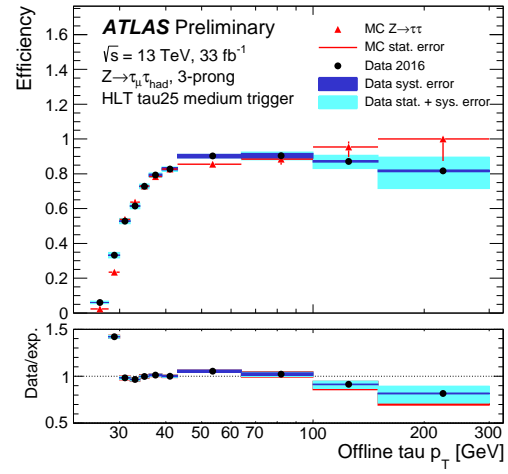

(b)

Figure 4: Comparison of the efficiencies of triggers used at the start of Run 2 in data and simulation, obtained using $Z \rightarrow \tau \tau$ events [4]. These triggers require a $p_{\mathrm{T}}$ of $25 \mathrm{GeV}$ at the calorimeter-only preselection, and $12 \mathrm{GeV}$ with isolation at L1. In (a) the efficiency on offline 1-prong candidates is shown, while (b) shows the same for offline 3-prong candidates.

BRT, while the identification of tau-leptons has been upgraded from a BDT to an RNN. The track multiplicity cut also changed, contributing to the improved performance at high pile-up. Combined with the developments in the hardware, such as the L1 calorimeter upgrade, tau-lepton triggers show a lot of promise for Run 3.

\section{References}

[1] ATLAS Collaboration. The ATLAS Experiment at the CERN Large Hadron Collider. JINST 3 (2008) S08003.

[2] ATLAS Collaboration. Cross-section measurements of the Higgs boson decaying into a pair of $\tau$-leptons in proton-proton collisions at $\sqrt{s}=13 \mathrm{TeV}$ with the ATLAS detector. Phys. Rev. D 99, 072001.

[3] ATLAS Collaboration. Search for direct stau production in events with two hadronic tau leptons in $\sqrt{s}=13 \mathrm{TeV}$ pp collisions with the ATLAS detector. ATLAS-CONF-2019-018.

[4] ATLAS Collaboration. The ATLAS Tau Trigger in Run 2. ATLAS-CONF-2017-061.

[5] ATLAS Collaboration. Performance of the ATLAS Trigger System in 2010. Eur. Phys. J. C 72 (2012) 1849.

[6] ATLAS Collaboration. Measurement of the tau lepton reconstruction and identification performance in the ATLAS experiment using pp collisions at $\sqrt{s}=13 \mathrm{TeV}$. ATLAS-CONF-2017-029.

[7] ATLAS Collaboration. Public Tau Trigger Plots for Collision Data. https://twiki.cern.ch/twiki/bin/view/AtlasPublic/TauTriggerPublicResults .

[8] ATLAS Collaboration. Identification of hadronic tau lepton decays using neural networks in the ATLAS experiment. ATL-PHYS-PUB-2019-033.

[9] ATLAS Collaboration. Level-1 Calorimeter Trigger Public Results. https://twiki.cern.ch/twiki/bin/view/AtlasPublic/L1CaloTriggerPublicResults . 